\title{
Growth and characterization of a new NLO material: L-serine sodium nitrate
}

\author{
Sd. Zulifiqar Ali Ahamed ${ }^{1}$, G. R. Dillip ${ }^{1}$, L. $\operatorname{Manoj}^{1}$, P. Raghavaiah ${ }^{2}$, B. Deva Prasad Raju ${ }^{* 1}$ \\ ${ }^{1}$ Department of Physics, Sri Venkateswara University, Tirupati - 517 502, India \\ ${ }^{2}$ School of Chemistry, University of Hyderabad, Hyderabad - 500 046, India
}

Received December 01, 2010; accepted December 17, 2010; published December 31, 2010

\begin{abstract}
Single crystals of L-serine sodium nitrate (LSSN), an organic nonlinear optical material have been synthesized by a slow evaporation technique at room temperature. The crystalline nature was confirmed by single crystal X-ray diffraction analysis. The powder Xray diffraction of a grown crystal was recorded and indexed. The chemical composition of grown crystals was determined by FTIR spectra. Optical absorption studies have shown that a UV cut off wavelength of the grown crystal is at $256 \mathrm{~nm}$ and it has a wide transparency window. The second harmonic generation (SHG) efficiency of the grown crystal was confirmed by the Kurtz and Perry powder technique.
\end{abstract}

Highly nonlinear materials are capable of fast and efficient processing of optical signals in the fast growing development of optical fiber communication systems. The nonlinear crystals have proved to be interesting candidates for a number of applications, such as second harmonic generation, frequency mixing, electro-optic modulation, optical parametric oscillation etc. [1]. Due to the technological importance of these nonlinear crystals the needs for high quality organic crystals have grown dramatically in the last decade. With rapid progress in the crystal growth technology, crystals having attractive nonlinear properties are being discovered. This has enabled the commercial development of single crystals with promising nonlinear optical properties. An organic molecule with significant nonlinear optical activity generally consists of a $\pi$-electron conjugated moiety substituted by an electron donor group on one end of the conjugated structure and an electron acceptor on the other end, forming a "push-pull" conjugated structure. The conjugated $\pi$-electron moiety provides a pathway for the entire length of conjugation under the perturbation of an external electric field. Functionalizing both ends of the $\pi$ bond system with an appropriate electron donor and acceptor group can increase the asymmetric electronic distribution in either or both the ground and excited state, thus leading to increased optical nonlinearity [2]. Organic molecules should have high second order hyperpolarizability $(\beta)$ to exhibit effective NLO properties in many cases, an order of magnitude smaller than the widely known inorganic materials [3].

*Email: drdevaprasadraju@gmail.com
L-serine is an organic compound under an amino acid category. It is one of the naturally occurring protenogenic amino acids. L-serine exists in a zwitterionic form; the molecule can combine with anionic, cationic and overall neutral constituents. In this paper we report the growth, structural, vibrational, optical and second harmonic generation (SHG) properties of single crystals of L-serine sodium nitrate (LSSN).

The starting material was synthesized by taking Lserine and sodium nitrate in a 1:1 stoichiometric ratio. The calculated amounts of these materials were dissolved in double deionized water of the $18.2 \mathrm{M} \Omega \mathrm{cm}^{-1}$ resistivity and the solution was filtered by a Whatman filter paper of the $11 \mu \mathrm{m}$. pore size. The prepared solution was allowed to dry at room temperature and the salts were obtained by a slow evaporation technique. The purity of the synthesized salt was further improved by a successive recrystallization process yielded a crystal of the $44 \times 12 \times 9 \mathrm{~mm}^{3}$ size in a growth period of 30 days. The grown single crystal is shown in Fig. 1.

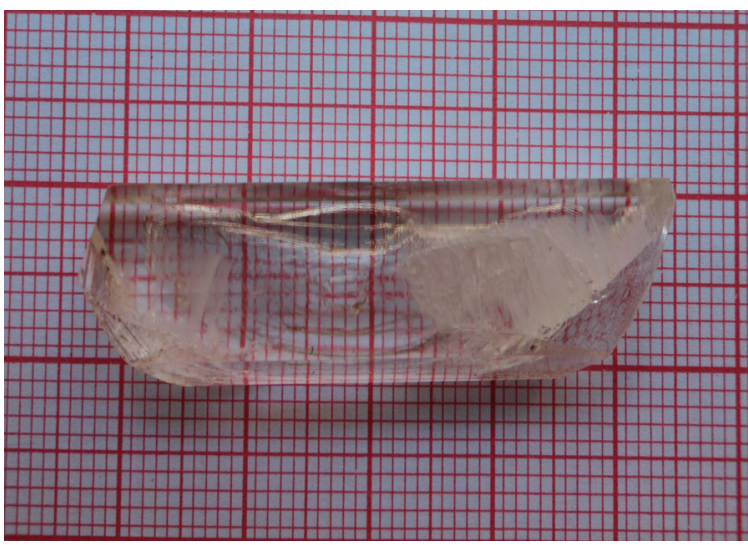

Fig. 1. The grown LSSN crystal.

\section{Single crystal $X$-ray diffraction}

Single crystal X-ray diffraction data recorded using an ENRAF-NONIUS MACH3 X-ray diffractometer with MoK $\alpha$ radiation $(\lambda=0.71073 \AA)$ to identify the structure of 
the grown crystal. The structure was solved by a direct method and refined by the full matrix least square technique using the SHELXL program. The calculated lattice parameters are presented in Table 1. It is observed from the XRD analysis that the LSSN crystal has an orthorhombic structure with the space group $\mathrm{P} 2{ }_{1} \mathrm{P} 2{ }_{1} \mathrm{P} 2{ }_{1}$.

\begin{tabular}{|l|l|}
\hline Unit cell dimensions & $\mathrm{a}=5.6268 \AA$ \\
& $\mathrm{b}=8.5988 \AA$ \\
& $\mathrm{c}=9.3677 \AA$ \\
& $\alpha=\beta=\gamma=90^{\circ}$ \\
Volume & $453.24 \AA^{3}$ \\
Crystal structure & Orthorhombic \\
Space group & $\mathrm{P} 2{ }_{1} \mathrm{P} 2{ }_{1} \mathrm{P} 21$ \\
Wavelength & $0.71073 \AA$ \\
Refinement method & Full matrix least square on $\mathrm{F}^{2}$ \\
$\mathrm{Z}$ & 4 \\
Density & $1.540 \mathrm{mg} / \mathrm{m}^{3}$ \\
\hline
\end{tabular}

Table 1. Single crystal XRD data of LSSN crystal.

\section{Powder X-ray diffraction analysis}

A powder X-ray diffraction study was carried out by employing a SIEFERT 3003 TT diffractometer with a characteristic $\mathrm{CuK} \alpha(\lambda=1.540598 \AA)$ radiation from $20^{\circ}$ to $60^{\circ}$ at a scan rate of $2 \% \mathrm{~min}$. The powder XRD pattern for the grown crystal is shown in Fig. 2. The appearance of sharp and strong peaks confirms good crystallinity of the grown sample. The observed values are in good agreement with the data available in the JCPDS card no: 27-1989. A slight shift in the sharp peak position at $22.93^{\circ}$ in the lower angle side may be due to the addition of sodium nitrate in LSSN crystal and it is also confirmed by a slight variation observed in the lattice parameters of the grown crystal.

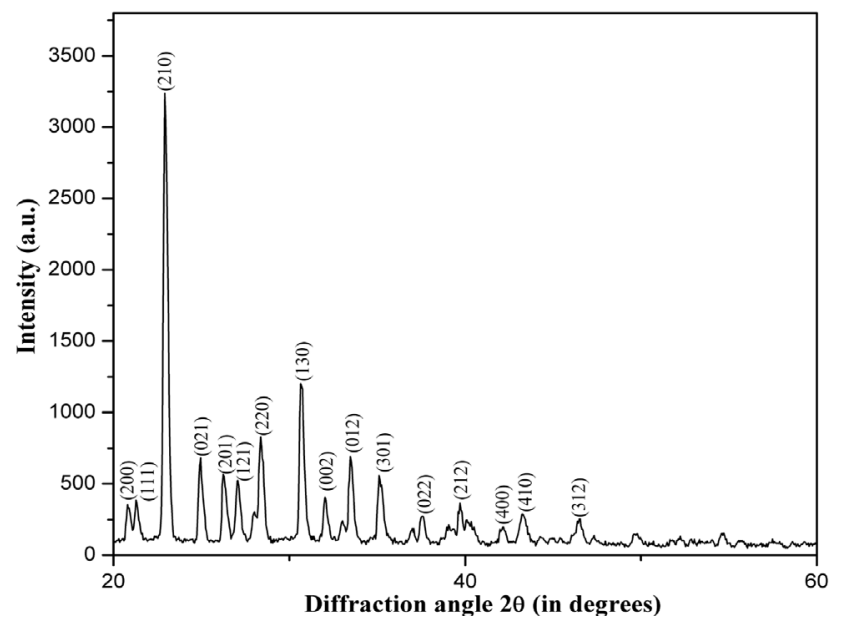

Fig. 2. Powder XRD pattern of LSSN crystal.

\section{Vibrational analysis}

The FTIR spectrum of LSSN crystal was recorded between $4000-450 \mathrm{~cm}^{-1}$ using a Perkin Elmer spectrometer by the $\mathrm{KBr}$ pellet technique. The resulting spectrum is shown in Fig. 3. The presence of $\mathrm{OH}$ is indicated by a broad absorption peak at $3461 \mathrm{~cm}^{-1}$. The frequencies observed at 3092 and $2727 \mathrm{~cm}^{-1}$ are attributed to $\mathrm{NH}_{3}{ }^{+}$ stretching and $\mathrm{C}-\mathrm{H}$ stretching. The absorption peaks observed at 1125 and $1013 \mathrm{~cm}^{-1}$ respond to $\mathrm{C}-\mathrm{N}$ group. The peaks observed at 918,610 and $525 \mathrm{~cm}^{-1}$ is attributed to $\mathrm{CH}_{2}$ group while the peak observed at $1600 \mathrm{~cm}^{-1}$ is attributed to $\mathrm{COO}^{-}$group. The presence of various functional groups of the grown crystal is in very good agreement with those reported in the literature [4].

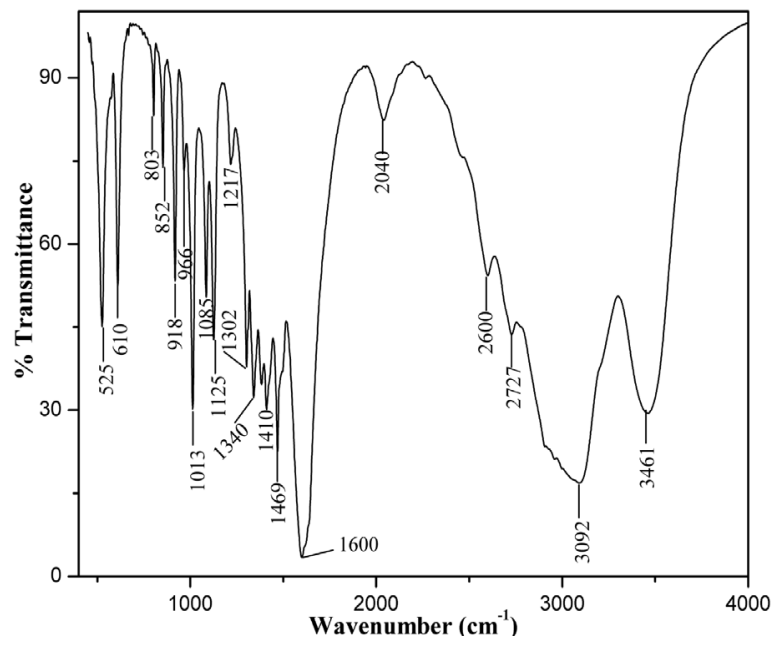

Fig. 3. FTIR spectrum of the grown crystal.

\section{Optical absorption}

The optical absorption spectrum of a good quality grown crystal was recorded in the wavelength range 2001000nm using a Perkin Elmer Lamda 935 UV-vis-NIR spectrometer. The obtained absorption spectrum is shown in Fig. 4, where the lower cut off region is obtained at $256 \mathrm{~nm}$. The UV spectra show the presence of a wide transparency window lying between $256 \mathrm{~nm}$ and $1000 \mathrm{~nm}$ with $\lambda_{\max }=256 \mathrm{~nm}$. From the relation $\mathrm{E}_{\mathrm{g}}=1.243 \times 10^{3} / \lambda_{\text {max }}$ the forbidden energy gap was estimated and is found to be $4.85 \mathrm{eV}$, which is a typical value of dielectric materials [5]. Hence, from the analysis of the absorption spectrum, it is evident that the grown crystal is transparent in the entire visible region without any absorption peak, which is the key requirement for any nonlinear optical crystal having applications in second harmonic generation (SHG). 


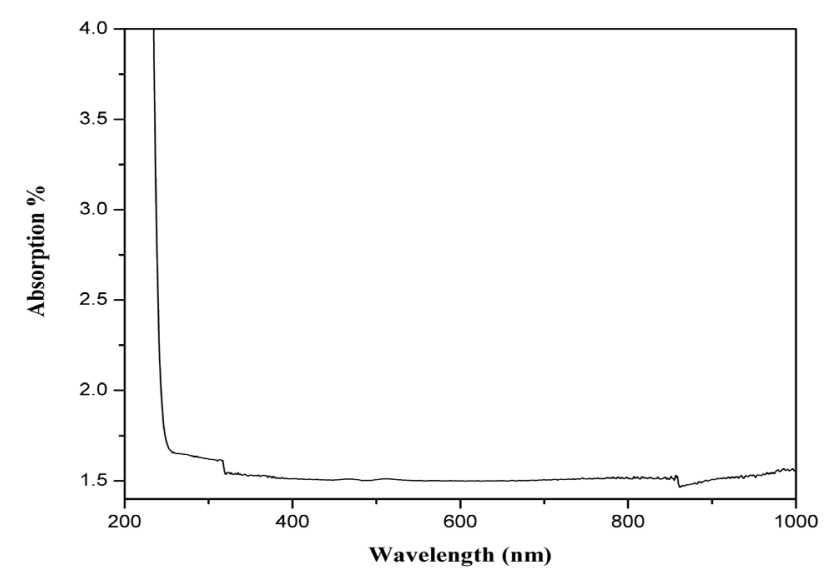

Fig. 4. Absorption spectrum of LSSN crystal.

\section{Nonlinear optical studies}

In order to confirm the NLO behaviour in the title compound, powdered samples were subjected to the Kurtz and Perry powder technique [6]. A Q-switched $\mathrm{Nd}$ :YAG laser beam of wavelength $1064 \mathrm{~nm}$ and $10 \mathrm{~ns}$ pulse width with an input rate of $10 \mathrm{~Hz}$ was used to test the NLO property of the sample. A grown crystal SHG signal of $6 \mathrm{mV}$ was obtained while the KDP gave an SHG signal of $15 \mathrm{mV}$ for input beam energy of $4.7 \mathrm{~mJ} / \mathrm{Pulse}$. The second harmonic signal generated in the crystalline sample was confirmed from the emission of green radiation from the crystal. Hence, it is used for applications in photonic and optoelectronic devices.
In conclusion, bulk size transparent single crystals of LSSN were successfully synthesized by a slow solvent evaporation method at ambient environment. The unit cell parameters were found by single crystal and powder XRD analysis and they agree well with the reported values. The presence of various functional groups of the grown crystal was confirmed by FTIR. The UV-vis-NIR absorption spectra showed that LSSN crystal was transparent in the entire visible region with a lower cut-off at $256 \mathrm{~nm}$, making it a potential candidate for NLO applications. The relative SHG conversion efficiency of the grown crystals is about 0.4 times higher than that of KDP sample, which indicates the suitability of LSSN crystals for photonic and NLO applications.

The authors are sincerely thankful to the SHG measurement facility extended by Prof. P.K. Das, Indian Institute of Science, Bangalore. The authors also acknowledge Sophisticated Analytical Instrument Facility (SAIF), Indian Institute of Technology, Chennai.

\section{References}

[1] D.S. Chemla, J.Zyss, Nonlinear optical properties of organic molecules and crystals (Academic Press 1987).

[2] H. Ringertz, Acta Crystallogr. B 27, 285 (1971).

[3] T. Pal, T. Karand, B. Gabriel, L. Rigi, Cryst. Growth Des. 4, 43 (2004).

[4] B.N. Moolya, A. Jayarama, M.R.S. Kumar, S.M. Dharmaprakash, J. Cryst. Growth 280, 581 (2005).

[5] M.M. Khandpekar, S.P. Pati, Opt. Comm. 283, 2700 (2010).

[6] S.K. Kurtz, T.T. Perry, J. Appl. Phys. 39, 3798 (1968). 